\title{
A cryogenic implant for producing reversible functional brain lesions
}

\author{
KENT G. SALSBURY and JAMES A. HOREL \\ State University of New York, Syracuse, New York
}

\begin{abstract}
This paper describes a permanently implanted device for applying cold to localized parts of the brain surface in order to produce reversible functional lesions in behaving animals. It consists of an array of several independently controlled stainless steel tubing loops, each shaped to fit a separate brain area. Cooled alcohol is pumped through the tubing and temperatures are monitored with attached thermocouples. Implanted loops can be cooled to $0^{\circ} \mathrm{C}$ within $15 \mathrm{sec}$, and temperatures can be maintained at $\pm 1^{\circ} \mathrm{C}$ of accuracy. Tissue tolerance is excellent: Implants have been demonstrated to last up to 1 year or more. Methods and materials for construction are discussed.
\end{abstract}

Cold has frequently been used to make reversible brain lesions in animal behavioral experiments (Fuster \& Alexander, 1970; Goldman \& Alexander, 1977; Hore \& Vilis, 1980; Skinner \& Lindsley, 1967; also see Brooks, 1983, for a review). There are many well-known advantages to the use of reversible lesions, a few of which are listed here. (1) Each animal can be used as its own control. (2) Experiments can be replicated in the same animals with identical lesions, and several different lesions can be made in the same animal. (3) Measurements of behavior can be made before, during, and after a lesion has been made. (4) Cold-produced lesions are made long after the effects of the surgical trauma have dissipated. (5) The function of brain areas can be suppressed during performance of parts of a task, provided that the task can be sufficiently spread out in time. The cryoloop described in this paper is a simple method for producing reversible lesions by temporarily withdrawing heat from the surface structures of the brain. Our recent experiments have concentrated on the temporal lobe of monkeys (Horel, Voytko, \& Salsbury, 1983; Voytko, Salsbury, \& Horel, 1982).

The cryoloop is an instrument designed to pass a very cold liquid (absolute methanol) through smallbore tubing loops that are closely applied to the brain surface. The temperature of the cryoloop depends upon the amount of cooled liquid pumped through it per unit of time. Thermocouples are used to monitor loop temperatures, and support and protection are provided by a stainless steel cylinder and plastic cap. All are cemented together into a compact, easily handled unit that can be permanently affixed to the skull. Connection and disconnection of tubing and

This work is supported by NINCDS Grant NS 1829-01. The authors wish to thank Nancy Snyder for typing the manuscript. The authors' mailing address is: Department of Anatomy, Upstate Medical Center, 766 Irving Avenue, Syracuse, New York 13210. thermocouple wires during experimental sessions are quickly accomplished. Animals exhibit no apparent discomfort from the apparatus itself or from the application of the cold.

Nine monkeys have been fitted with cryoloops by this method thus far. Two were sacrificed after 8 months due to the malfunction of thermocouples made with fine-gauge wire $(0.077 \mathrm{~mm}$ diam). One monkey was sacrificed after 7 months of testing because infection around the skull attachments caused the probes to become loose. Two monkeys with similar but smaller probes were sacrificed after 9 and 12 months when their experiments were terminated. Of the remaining animals, the monkey with the longest implant (9 months) and the other monkeys are healthy and continue to perform well.

Pilot experiments demonstrated that clear reversible behavioral effects are elicited with temperatures of $0^{\circ} \mathrm{C}$ as measured on the metal of the loop itself. With the system described here, this target temperature can be achieved in less than 15 sec on implanted cryoloops. Our usual procedure is to use a more gradual cool-down of $30 \mathrm{sec}$, which avoids overshooting the $0^{\circ} \mathrm{C}$ point. Once established, the target temperature can be maintained at about $\pm 1^{\circ} \mathrm{C}$ of the target temperature through careful control of the pump rate. The average time for the loop to return to the normal body temperature of approximately $37^{\circ} \mathrm{C}$ after the pump is switched off varies somewhat with the time cooled, but it is about $3 \mathrm{~min} 15 \mathrm{sec}$ for a cooling period of $5 \mathrm{~min}$. Warm-up is rapid at first, reaching $20^{\circ} \mathrm{C}$ within $23 \mathrm{sec}$, but proceeds more gradually after that.

Ongoing experiments on the spread of the cold indicate a steep temperature gradient around the loops. A thermocouple placed in the tissue adjacent to the loop tubing but across the dura measured $\pm 12^{\circ} \mathrm{C}$ in a behaving monkey when that loop was cooled to $0^{\circ} \mathrm{C}$. Thermocouples a few millimeters away were 
cooled only a few degrees. Our results appear to be consistent with those of other experimenters as reviewed by Brooks (1983).

The loops that administer the cold are made from 23-ga (0.635-mm-O.D., 0.33-mm-I.D.) hypodermic stainless steel tubing. This size was chosen because the Teflon tubing (1.5 mm O.D., $0.5 \mathrm{~mm}$ I.D.) carrying the cooled methanol to and from the unit simply can be pushed onto the ends of the stainless steel tubing to produce a snug, leak-proof connection capable of withstanding fluid pressures up to the maximum of the fluid pump (rated at $100 \mathrm{psi}$ ). The stainless steel tubing is bent with a round nose pliers into the desired loop shape and size. Those of our most recent experiments were oblong loops of $10 \times 3 \mathrm{~mm}$ I.D., four on each side of the brain (Figure 1). With practice, loops of less than $2 \mathrm{~mm}$ in diameter can be made. Smaller loops are possible with smaller sized tubing. Thin-wall tubing, which allows a greater fluid flow per unit of time at a given pressure, can also be used. The disadvantage is that it readily dents and crimps during manipulation. To avoid this, it may be first filled with a low-meltingpoint $\left(<100^{\circ} \mathrm{C}\right)$ bismuth alloy (Small Parts, Inc.), bent, and then flushed out under boiling water. We

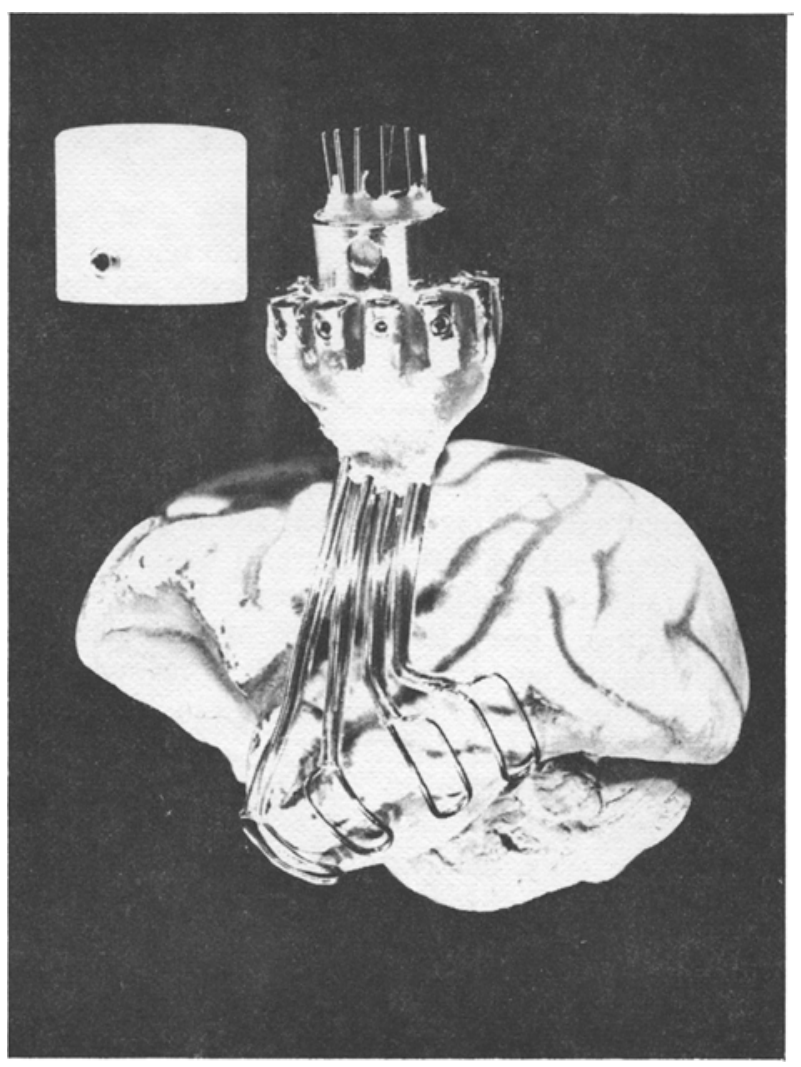

Figure 1. Cryoloop and monkey (Macaca fascicularis) brain cast. prefer to use hypodermic tubing, which is more easily handled, and just increase the pump rate in order to achieve our normal testing temperatures of $0^{\circ} \mathrm{C}$.

Cryoloop temperatures are measured by Bailey BAT-9 and BAT-12 direct-reading thermometers connected to thermocouples soldered between the input and exit sides of the stainless steel tubing leading to the loop as close to the loop itself as possible. Matched thermocouple wire composed of copper and constantan $(\mathrm{Cu} / \mathrm{Co})$ is used here because of its accuracy of response across our operating temperatures. The thermocouples are made by twisting the bare ends of the paired wire together, trimming them off closely, and soldering. This is done simultaneously with the soldering of the thermocouple to the loop tubing; a stainless steel solder and flux are used. We now use 30-AWG-ga $(0.254-\mathrm{mm})$ Teflon insulated $\mathrm{Cu} / \mathrm{Co}$ thermocouple wire for the thermocouples and leads. Thermocouple wire of $0.077-\mathrm{mm}$ diameter or smaller may be used, but often has a shorter performance life once in place, possibly because of flexion of the wire due to differential expansion and contraction of the apparatus during temperature changes. Thermocouples, lead wires, and stainless steel tubing are ensheathed up to, but not including, the loop with heat-shrink Teflon tubing (expanded inner diameter of $1.70 \mathrm{~mm}$ ). This holds the wires in place and protects the tissue from the toxic metal of the thermocouple. The lead wires are soldered to $7 \times 1.3 \mathrm{~mm} \mathrm{Cu} / \mathrm{Co}$ spades cut from commercially available spade connectors (Omega Engineering, Inc.). The spades are inserted into special Epoxylite insulated stainless steel connector fittings (Figure 2). The connector fittings are designed so that paired 26-AWG-ga $(0.4-\mathrm{mm}) \mathrm{Cu} / \mathrm{Co}$ thermocouple wire extending to the thermometer may be slipped into the end of a pair of fittings and tightened against the connector spade of each with a small setscrew. This configuration produces a quick-connect paired connector of approximately $7 \times 7 \times 3 \mathrm{~mm}$ for each thermocouple and allows several to be mounted on an animal's head at once. A thermocouple switchbox permits monitoring of multiple thermocouples for each thermometer.

The exposed lead ends of the cryoloops are protected by a stainless steel cylinder and a Delrin cap (Figure 3). The stainless steel tubing leads of the loops are cemented within the cylinder with craneoplastic cement (Plastic Products Co.), and the thermocouple connectors are cemented to the outside. The lead wires pass through slots in the cylinder. Cryoloop lead ends are color coded by imbedding colored wire insulation inside the cylinder around the input side of the loop; the input side of the loop is that side of the loop that is most anterior in each case. Thermocouple connectors are arranged around the cylinder in their respective anterior-posterior order, thus leaving a space on the medial side of the 


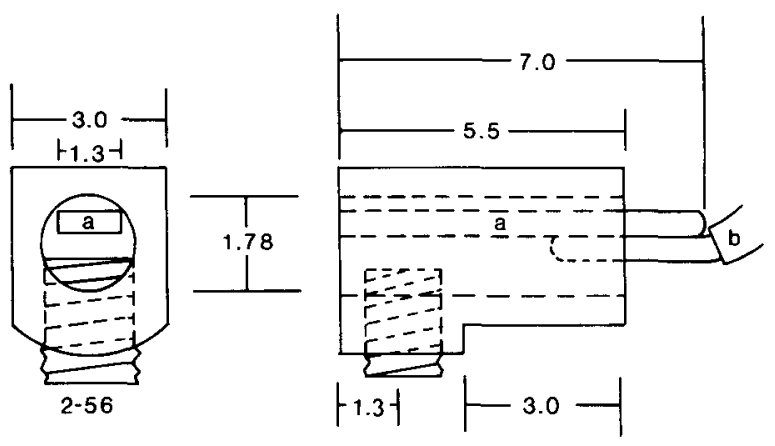

Figure 2. Thermocouple connector fitting. Measurements are in millimeters. (a) Copper or constantan spade; (b) thermocouple wre.

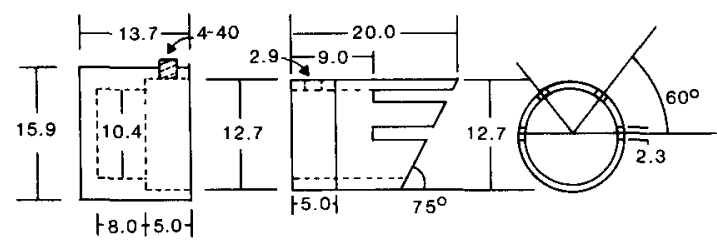

Figure 3. Protective cylinder and cap. Measurements are in millimeters.

cylinder between the most anterior and most posterior fittings. The "skull end" of the cylinder is cut at an angle to conform to the angle of the skull at the mounting site. The cap is held in place by a 2-56 setscrew.

Once cemented in place, the loops and leads are bent to conform to the shape of the brain surface. A plaster brain cast is used as the template (Figure 1). The cast is made from a flexible mold of a fixed brain taken from an animal the same size as the test animal. Alginate Elastic dental-impression powder is the mold medium, and Whip Mix model plaster provides the cast material. Several casts may be poured from a single mold, and molds may be stored for months if wrapped and refrigerated.

The completed cryoloop unit may be thoroughly tested for reliability before surgery. It is best to sterilize with gas. Each right and left cryoloop unit is inserted as one piece through a small craniotomy barely large enough to permit the entry of the loop system. The loops are inserted with visual guidance between the bone and the dura. Placement below the dura is possible, but increases the possibility of cortical damage. Each unit is cemented in place with cranioplastic cement surrounding a few $0-80 \times 1 / 8$ in. binding-head machine screws that have been threaded into hand-drilled holes in the skull.

An initial investment of approximately $\$ 200$ in materials is required for basic cryoloop construction. Our cost is less than $\$ 50$ per completed cryoloop unit, most of which cost is shop labor for the construction of cylinders, caps, and thermocouple connectors.

Figure 4 shows the system by which cooled methanol is delivered to the cryoloop. Absolute methanol is drawn from the reservoir flask by a synchronous rotating and reciprocating piston pump (Fluid Metering, Inc. Model RP-SY-1CSC with low-flow adapter) through a $10-\mathrm{mm}$ pump inlet filter and $3.0 \mathrm{~mm}$ O.D. $x 1.5 \mathrm{~mm}$ I.D. Teflon tubing. It is then pumped via $1.5 \mathrm{~mm}$ O.D. $\times 0.8 \mathrm{~mm}$ I.D. Teflon tubing through a coil submerged in a $-78.4^{\circ} \mathrm{C}$ bath of methanol and crushed dry ice. Upon exiting from the bath, the 0.8-mm-I.D. tubing connects by an Altex Teflon connector to $1.5 \mathrm{~mm}$ O.D. x $0.5 \mathrm{~mm}$ I.D. tubing. This Teflon tubing leads to the animal's head and connects to the input side of the cryoloop, as described earlier. Tubing of the same size is used to carry the methanol away from the cryoloop. It connects to 0.8-mm-I.D. tubing again, which leads back to the reservoir for reuse.

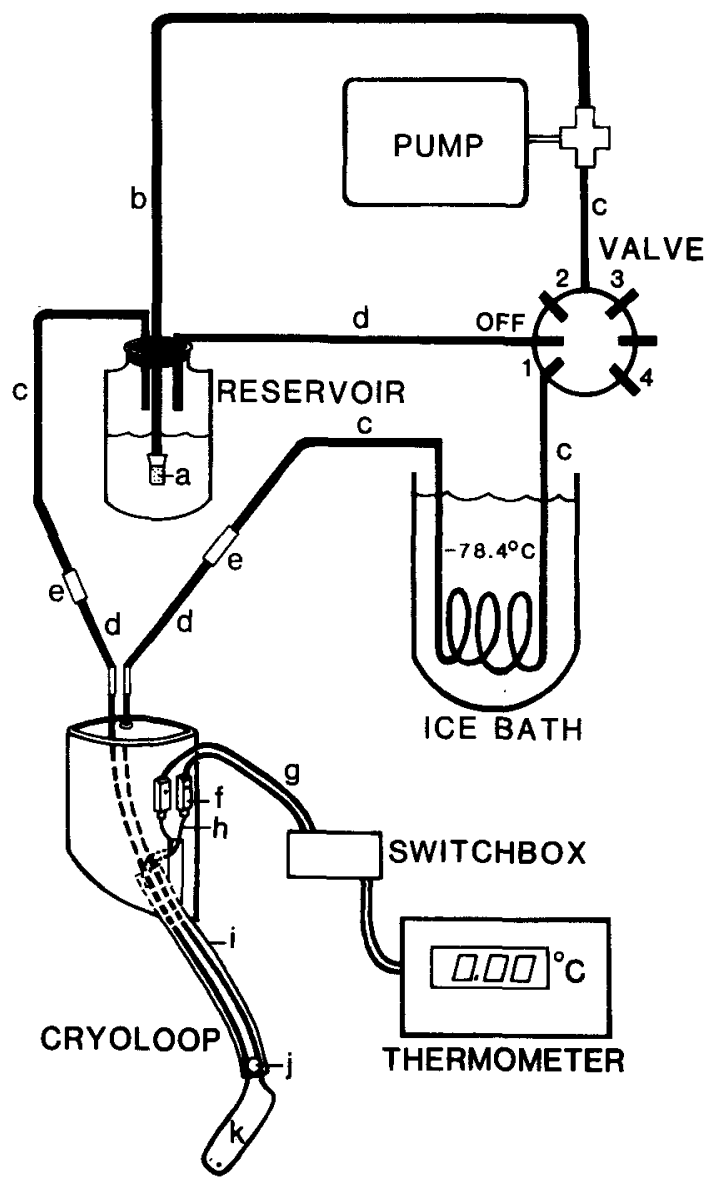

Figure 4. The coolant delivery system: (a) Pump inlet filter; (b) 1.5-mm-I.D. Teflon tubing; (c) 0.8-mm-I.D. Teflon tubing; (d) 0.5-mm-I.D. Teflon tubing; (e) tubing connector; (f) thermocouple connector; (g) 26-AWG thermocouple wire; (h) 30 AWG thermocouple wire; (i) heat-shrink Teflon-tubing sheath; (j) thermocouple; (k) loop. 
A valve inserted into the line between the pump and the cold bath provides control over which loop, of several that are in place, is cooled. The "off" position is really a shunt of 0.5 -mm-I.D. tubing that leads back to the reservoir. This allows the pump to remain on at its set flow-rate position throughout the course of the experiment.

Our present setup includes two separate systems of pumps, tubing, valves, and reservoirs for accurate equalization of temperature between the right and left pairs of cryoloops. Six-position Rheodyne Type 50 Teflon rotary valves (Rainin Instruments, Inc.) for each system provide instant control of multiple cooling coils and cryoloops. Additional Teflon fittings (Altex, Omnifit, etc.) allow for a variety of cooling combinations and sequences.

The cryoloop has the advantage of being adaptable in size and shape to the brain structure being studied. It can also be used to cool the depths of the sulci by removing the cortex of one bank of the sulcus and placing the loop over the pia that folds into the sulcus (Horel, 1983). The cryoloop also can be used to study subcortical areas by surrounding the loop leads with heater coils (Skinner \& Lindsley, 1968). Although the method described herein was designed for use in monkeys, it should be equally applicable to animals with much smaller brains.

\section{REFERENCES}

Brooks, V. B. Study of brain function by local reversible cooling. Reviews of Physiology, Biochemistry and Pharmacology, 1983, 95, 1-109.

Fuster, J. M., \& Alexander, G. E. Delayed response deficit by cryogenic depression of frontal cortex. Brain Research, $1970,20,85-90$.

Goldman, P. S., \& Alexander, G. E. Maturation of prefrontal cortex in the monkey revealed by local reversible cryogenic depression. Nature, 1977, 267, 613-615.

HorE, J., \& Vilis, T. Arm movement performance during reversible basal ganglia lesions in the monkey. Experimental Brain Research, 1980, 39, 217-228.

HoREL, J. A. The effects of local cooling of inferotemporal cortex on the performance of visual tasks. Society for Neuroscience Abstracts, 1983, in press.

Horel, J. A., VoytKo, M. L., \& Salsbury, K. G. Visual learning suppressed by cooling the temporal pole. Behavioral Neuroscience, 1983 , in press.

Skinner, J. E., \& Lindsley, D. B. Electrophysiological and behavioral effects of blockade of the nonspecific thalamo-cortical system. Brain Research, 1967, 6, 95-118.

Skinner, J. E., \& Lindsley, D. B. Reversible cryogenic blockade of neural function in the brain of unrestrained animals. Science, 1968, 161, 595-596.

VoytKo, M. L., Salsbury, K. G., \& Horel, J. A. Learning but not retention affected by cooling anterior temporal lobe. Society for Neuroscience Abstracts, 1982, 8, 313.

(Manuscript received May 26, 1983; revision accepted for publication July 13, 1983.) 